STUDI

FRANCESI

\section{Studi Francesi}

Rivista quadrimestrale fondata da Franco Simone

170 (LVII | II) | 2013

Varia

\title{
Valentin et Orson. An Edition and Translation of the Fifteenth-Century Romance Epic, Edited and translated by Shira Schwam-Baird
}

\section{Maria Colombo Timelli}

\section{(2) OpenEdition \\ Journals}

Édition électronique

URL : https://journals.openedition.org/studifrancesi/3032

DOI : 10.4000/studifrancesi.3032

ISSN : 2421-5856

Éditeur

Rosenberg \& Sellier

\section{Édition imprimée}

Date de publication : 1 juillet 2013

Pagination : 437-438

ISSN : 0039-2944

\section{Référence électronique}

Maria Colombo Timelli, « Valentin et Orson. An Edition and Translation of the Fifteenth-Century Romance Epic, Edited and translated by Shira Schwam-Baird », Studi Francesi [En ligne], 170 (LVII | II) | 2013, mis en ligne le 30 novembre 2015, consulté le 31 janvier 2023. URL : http://journals.openedition.org/ studifrancesi/3032 ; DOI : https://doi.org/10.4000/studifrancesi.3032

Ce document a été généré automatiquement le 31 janvier 2023.

\section{c) (†) $\odot$}

Creative Commons - Attribution - Pas d'Utilisation Commerciale - Pas de Modification 4.0 International - CC BY-NC-ND 4.0

https://creativecommons.org/licenses/by-nc-nd/4.0/ 


\title{
Valentin et Orson. An Edition and Translation of the Fifteenth-Century Romance Epic, Edited and translated by Shira Schwam-Baird
}

\author{
Maria Colombo Timelli
}

\section{RÉFÉRENCE}

Valentin et Orson. An Edition and Translation of the Fifteenth-Century Romance Epic, Edited and translated by Shira Schwam-Baird, Tempe, Arizona, ACMRS, 2011, pp. 543.

1 Transmis uniquement par des imprimés, Valentin et Orson est un long récit épique en prose qui a joui d'un remarquable succès d'édition, en France, Allemagne, Angleterre, tout au long $\mathrm{du} \mathrm{XVI}^{\mathrm{e}}$ siècle et bien au-delà, jusqu'aux éditions d'Épinal. Malgré une longévité qui aura duré plus de trois siècles, cette histoire aventureuse de deux frères jumeaux, fils de la sœur de Pépin le Bref, n'avait connu jusqu'ici aucune édition scientifique, et les quelques études parues encore récemment se basaient nécessairement sur les imprimés anciens: c'est une lacune à laquelle Shira SchwamBaird aura porté remède, bien que son édition - basée sur l'editio princeps, parue en 1487 - ne réponde pas entièrement aux critères d'une édition «critique» au sens plein du mot.

2 L'introduction (pp. IX-XXXII) rappelle d'abord l'hypothèse d'une source en vers, avancée par G.E. Klemming en 1846 et adoptée par G. Doutrepont en 1939, fondée essentiellement sur l'existence d'une rédaction versifiée en moyen bas allemand, Valentin und Namelos, de la première moitié $\mathrm{du} \mathrm{xv}^{\mathrm{e}}$ siècle. Suit une discussion sur le «genre» de l'œuvre, qui conjugue des thèmes et motifs propres de la chanson de geste avec d'autres ramenant indiscutablement vers le roman chevaleresque: d'autre part, non seulement la forme-prose tend à brouiller les genres littéraires du moyen âge 
classique, mais - comme l'a souligné M. Zink - cette hybridité même semble être à l'origine du grand succès de certains récits dans la longue durée (on rappellera entre autres le cas de La Belle Hélène de Constantinople). L'éditrice présente ensuite brièvement l'incunable et les gravures qui l'illustrent, dont on trouvera la description détaillée en annexe (pp. 535-537). Les principes d'édition et de traduction sont exposés rapidement aux pp. XXIII-XXv. L'étude littéraire aborde deux questions: les sources folkloriques du récit et le motif de l'homme sauvage, dont Orson représente une réalisation littéraire particulièrement réussie.

3 Le texte édité est donc celui fourni en 1487 par l'imprimeur lyonnais Jacques Maillet, auquel on doit d'autres éditions de romans «médiévaux»: outre Baudouin de Flandre, deux œuvres de Raoul Le Fèvre, Jason et Médée et le Recueil des Histoires de Troye. L'absence d'un texte de contrôle - l'apparat en bas de page enregistre uniquement les fautes de l'incunable, la présence de bois gravés et la foliotation ancienne - nous paraît regrettable, dans la mesure où le recours à l'édition fournie en 1495, à Lyon toujours, par Jacques Arnoullet aurait permis de reconnaître et de corriger un nombre important de fautes. Par ailleurs, l'éditrice ne signalant pas les changements de colonne, le repérage d'une leçon dans les pages denses de l'incunable devient malaisé.

4 La transcription est dans l'ensemble assez fiable, même si des erreurs demeurent. Nous n'en citerons que quelques-unes à titre d'exemple: «ce que fol pense il demeure estre parfait» (p. 24, sic au f. a7ra; le célèbre proverbe «moult remaint de ce que fol pense» n'est évidemment pas reconnu, mais la leçon correcte, imparfait, se lit chez Arnoullet); «Il [l'archevêque de Constantinople] laisa rochet et aumusce et comme irregulier et apostat a sainté son espee» (p. 34 de l'éd., lire: sainte); «... tant que par divin miracle son enfant luy fera rendu» (p. 40 de l'éd., lire: sera); «[Orson] devint grant en peu de temps et commença a fraper les aultres de la forez tant que toutes le doubtoient...» (p. 46; la lacune au f. b4rb aurait pu être remplie grâce à Arnoullet: les aultres <bestes> de la f.); "Par le conseil mauvais de l'archevesque furent elevé plusieurs nouvelles coustumes...», titre du ch. 7, f. b4vb, où l'adverbe introducteur Comment doit être intégré (p. 48; leçon correcte chez Arnoullet); "[les messagiers] sont venus a Romme devers le roy Pepin lequel ilz saluerent et luy en fait reverence...» (éd. p. 56; ont au f. b7ra); «l'empereur le fit chevalier et lui donna la colle en lui promettant donner villes, chasteaulx, et grandes richesses...» (p. 59, sic au f. b8ra; collee chez Arnoullet); «[l'archevesque] perdit son escu et la le marchant le getta hors des licices afin qu'il ne s'en peust plus ayder» (p. 64, à corriger évidemment en lices, à plus forte raison que la faute s'est produite au f. c1ra lors d'une division en syllabes en fin de ligne, li/cices; lices se lit bien chez Arnoullet). Une liste serait certainement fastidieuse; répétons cependant que des vérifications dans un texte de contrôle auraient éclairci pas mal de passages obscurs, tels que les deux qui suivent: "Car aultrement venger ne nous en pourrons du tout a nostre plaisir le royaulme gouverner sans nul contredit» (p. 86, sic au f. c6rb-va), où un saut du même au même s'est produit (cf. Arnoullet: «Car a. venger ne nous en pourrions, et lors pourrions du tout a nostre p. ...»); «Tu as droit, dit le roy. Or t'en va en ma court et may a pris la perte car [ta femme] rendue te sera» (p. 86 toujours, sic au f. c6vb; Arnoullet: «Or t'en va en ma court et metz a pris la perte»).

5 Des corrections s'avèrent en revanche superflues. Il en est ainsi p. 18, où Pépin s'adresse à Bellissant en lui disant: «Belle seur, souviengne vous du lieu dont vous estes yssue...» (f. a5vb, même leçon chez Arnoullet; S.S.-B. intervient sur la forme impersonnelle en corrigeant «souviengnez vous»). 
Le texte est accompagné d'une traduction anglaise en regard (page de droite), sur laquelle je n'oserai pas m'exprimer, mais qui semble établie avec soin.

7 Deux appendices: la première (pp. 531-534) fournit la liste de 50 éditions de Valentin et Orson, parues entre 1489 et 1846; la seconde, comme on l'a dit, donne la description des bois gravés: celui qui illustre le f. 1v est reproduit sur la couverture du livre. La bibliographie (pp. 539-543) sépare les «Primary sources» (quelques incunables et éditions $\mathrm{du} \mathrm{XVI}^{\mathrm{e}}$ siècle, ainsi que des éditions modernes, entre autres celles des autres versions médiévales de l'œuvre) et les sources secondaires. Signalons aussi que, après la parution de son édition, Shira Schwam-Baird a rédigé la notice de ce texte pour le nouveau répertoire des «mises en prose» partiellement en ligne sur le site «http:// users2.unimi.it/lavieenproses/», à paraître dans les Classiques Garnier; dans celle-ci la liste des éditions anciennes est ultérieurement enrichie et corrigée et la bibliographie critique complétée.

En conclusion, même en l'absence de quelques compléments essentiels (aucune étude linguistique, pas de glossaire, ce qui s'explique sans doute par la présence de la traduction anglaise en regard), l'édition de Shira Schwam-Baird aura le mérite de mettre enfin à la disposition des lecteurs un texte agréable, amusant par traits, que la critique a tout intérêt à (re)découvrir. 